\title{
Determination of view vectors from image warping mapping functions
}

\author{
David S. Nobes \\ Cranfield University \\ School of Engineering \\ Optical Sensors Group \\ Cranfield, Bedfordshire, MK43 OAL, United \\ Kingdom \\ Bernhard Wieneke \\ LaVision $\mathrm{GmbH}$ \\ Anna-Vandenhoek-Ring 19 \\ D-37081 Göttingen, Germany \\ Ralph P. Tatam, MEMBER SPIE \\ Cranfield University \\ School of Engineering \\ Optical Sensors Group \\ Cranfield, Bedfordshire, MK43 OAL, United \\ Kingdom \\ E-mail: r.p.tatam@cranfield.ac.uk
}

\begin{abstract}
The measurands of several reported laser-based measurement techniques are sensitive to both the propagation direction of the laser and the viewing direction from the region of interest to the detector. For such imaging techniques, the view vector must be determined uniquely for each pixel in the detector array. The bulk view vector is often physically measured and a simple model used to determine the view vector for each pixel. This, however, has limitations where access is limited, the distances involved are small, or the optical system employed introduces errors. We describe a procedure to determine the unique view vector from a planar region to the detector (CCD camera) for each element in a 2-D array based on a reference target aligned with the planar region of interest. Determination of the view vector is based on the spatial distribution of the mapping function used to dewarp the view. No physical measurement of the view vector is required. Good agreement is achieved when the procedure is compared to a simple pin-hole camera model of the view using a computed test target. (c) 2004 Society of PhotoOptical Instrumentation Engineers. [DOI: 10.1117/1.1636765]
\end{abstract}

Subject terms: view angle determination; image warping; planar Doppler velocimetry; machine vision; three-component measurement.

Paper 020458 received Oct. 18, 2002; revised manuscript received Mar. 27, 2003, and Jul. 25, 2003; accepted for publication Aug. 7, 2003.

\section{Introduction}

There are several laser-based measurement systems where the measurand is a function of the relative angle between the incident laser illumination and the view vector from the measurement volume to the detector. Examples of these techniques include planar Doppler velocimetry, ${ }^{1,2}$ (PDV) electronic speckle pattern interferometry, ${ }^{3}$ (ESPI) and shearography. ${ }^{4}$ Accurate determination of this angle, or more specifically the view vector, is important in achieving accurate measurements. This can be highlighted by considering the fundamental principle of the flow measurement technique, PDV. In this technique, the Doppler-shifted laser light that is scattered off particles that have been seeded into a flow is measured using a frequency-to-intensity converter. The laser used in the PDV technique is frequency tuned onto the side of a single absorption line of molecular iodine, which is used as an absorption line filter to attenuate the signal based on the frequency of the scattered light. Once the Doppler shift, $\Delta \nu$ is determined, the Doppler formula, $\Delta \nu=\lambda^{-1}(\hat{o}-\hat{i}) \cdot \mathbf{V}$ can be used to determine the velocity of the particles and hence infer the velocity of the flow. Here, $\lambda$ is the wavelength of the illumination laser, $\hat{o}$ is a unit vector describing the direction from the measurement volume to the detector, $\hat{i}$ is the propagation direction unit vector of the laser, and $\mathbf{V}$ is the velocity of the scattering medium. The vector sum, $(\hat{o}-\hat{i})$ describes the direction of the component of $\mathbf{V}$ that the measurement is sensitive to and is termed the sensitivity vector of the measurement system. It is clear from the Doppler formula that multiple components of the velocity can be determined by using multiple views of the same measurement volume. Determination of $\hat{o}$ is thus important not only for the accuracy of the measurement but also in the determination of multicomponents.

Measurement of $\hat{o}$ can be determined by physically measuring the vector from the measurement volume to the detector and then normalizing the vector. This is standard practice for most PDV systems reported in the literature. ${ }^{1}$ However, this method has some limitations. The view vector $\hat{o}$, as shown in Fig. 1, is the bulk unit view vector for the system and this is only true for the central pixel of the 2-D array of a CCD camera. Each individual pixel $(i, j)$ in the array views a different region of the plane under investigation and hence has a unique view vector $\hat{o}_{(i, j)}$. This view vector is a function of the area viewed, the physical size of the array and the distance between the array and the viewed area. Accurate calculation of the view vector $\hat{o}_{(i, j)}$ for each individual pixel $(i, j)$, in the camera array is an important part of the full data processing procedure. Consider a configuration where a camera, $1 \mathrm{~m}$ from a plane is viewing a region of $100 \times 100 \mathrm{~mm}$ and is viewing perpendicularly. The variation in the view vector across the field is $\sim \pm 6 \mathrm{deg}$. This would results in an error of $\sim 5 \%$ in the computed velocity if there is no correction for view angle.

Direct measurement of the bulk view vector is only suitable for situations where the distances involved are large, so that any measurement error does not introduce a significant error in the vector direction. After $\hat{o}$ is determined, a correction to the view vector for each individual pixel can be achieved by assuming a simple pin-hole camera model ${ }^{5}$ 


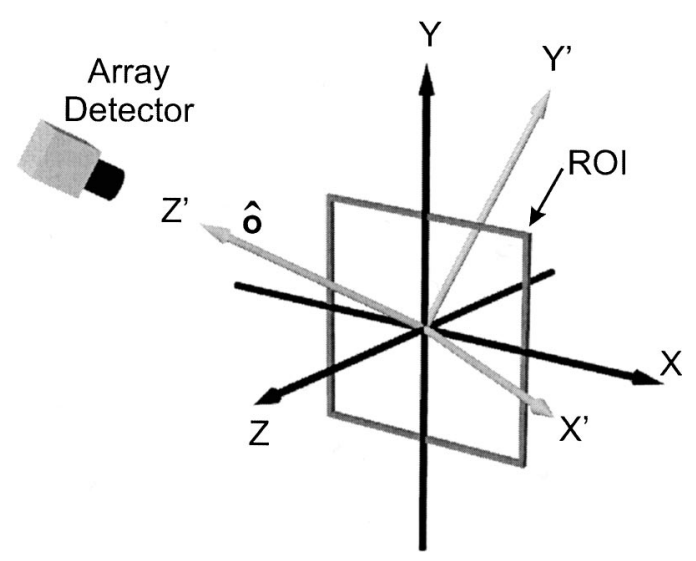

Fig. 1 Relationship of the Cartesian coordinate system of the experiment to the orthogonal coordinates system seen by the detector and the region of interest (ROI) viewed by the detector. The direction of the $Z^{\prime}$ axis is aligned with the bulk view vector $\hat{o}$.

of the view and correcting accordingly. Direct measurement of the bulk view vector is also difficult and potentially error prone in situations of restricted physical access or where the dimensions are small. Any error associated with the measurement of the bulk view vector will propagate through the calculation of all other view vectors. Use of the pin-hole model of the view also limits the accuracy of the measurement of $\hat{o}_{(i, j)}$ by assuming that there are minimal optics used in the collection of the image and that the optical train does not significantly alter the view vector, through distortion of the image.

There is therefore a need for an algorithm that can determine $\hat{o}_{(i, j)}$ for each individual pixel that does not rely on physical measurement. Several techniques are presented in the literature that have been developed for the calibration of camera parameters. These camera calibration procedures determine the bulk camera intrinsic (i.e., focal length) and extrinsic (i.e., camera position) parameters and include methods that use a single view of a target from a single camera, ${ }^{6}$ multiple views of a target from a single camera, 6,7 and multiple views from a number of cameras. ${ }^{5}$ The procedures process images of test targets or images that have know points located within the field of view. A limitation of these techniques is that only bulk parameters are calculated, hence only the bulk view vector $\hat{o}$ is found. Individual correction of each pixel for view angle is again needed after the bulk parameters are derived.

Techniques such as PDV are unique in that they measure over a plane defined by the propagation of the expanded laser beam into a sheet. For multicomponent measurement, multiple views of the region of interest (ROI) are collected and mapped onto a single coordinate system oriented with the experiment. ${ }^{1,2}$ This requires the dewarping of individual views onto the predefined plane and enables the interpolation of different measured components onto the same spatial grid. A calibration procedure used to determine the dewarping mapping functions using a test target of coplanar location markers that are at a known spacing is often employed. ${ }^{1,2}$ The dewarping procedure corrects the view for image perspective distortion as well as radial distortion and aberrations introduced by optical components within the imaging system. The mapping function therefore carries information about the bulk view vector to the target as well as for each pixel in the array and information about any local change in the view vector introduced by local distortion of the know target image.

In the remainder of this paper a procedure is developed to determine the view vector $\hat{o}_{(i, j)}$ for individual pixels from the mapping function determined in a dewarping procedure and the derived scaling information from a test target. The procedure is then tested against a computergenerated test target, where the view is at a known angle to the target plane.

\section{Dewarping Images of the ROI}

The ROI investigated by planar techniques is a region defined by the expansion of the laser beam into a sheet. A target, overlaying the laser sheet, consisting of a regular array of location marks is used to define the field of view and allow collection of a calibration image used to dewarp the view. The ROI imaged by the camera is a plane overlapping the $X-Y$ plane of a Cartesian coordinate system that is oriented to the experiment, as shown in Fig. 1. The positive $Z$ axis of this coordinate system is oriented toward the camera so that the location of the camera is in positive $Z$ space. If the camera were viewing perpendicular to the ROI with its optical axis pointing at the origin, the camera's optical axis, and hence it's bulk view vector $\hat{o}$ would coincide with the $Z$ axis. For any viewing angle away from the normal, the captured image would be a perspective view of the ROI. A second orthogonal coordinate system $\left(X^{\prime}, Y^{\prime}, Z^{\prime}\right)$ is aligned with the view direction of the camera such that the $Z^{\prime}$ axis is always aligned with the optical axis of the camera. In this scenario, the bulk unit view vector $\hat{o}$ of the camera aligns with the $Z^{\prime}$ axis.

A computed mapping function can define the transformation from the ideal rectilinear "corrected" plane to the distorted plane as recorded by the camera using

$x^{\prime}=M_{x}(x, y)$,

$y^{\prime}=M_{y}(x, y)$,

where the true "corrected" coordinates are given by $x$ and $y$, while the coordinates in the observed distorted image plane are given by $x^{\prime}$ and $y^{\prime}$, and $M_{x}$ and $M_{y}$ are the mapping functions that are determined by a dewarping algorithm. To enable mapping onto a predefined grid size and scaling, the mapping of $x^{\prime}$ to $x$ is achieved via an intermediary coordinate system $(s, t)$, which enables the mapping of several views onto the same grid size and subsequent scaling. This is a linear mapping of $s(x)$ and $t(y)$ to obtain scaling information. The mapping can thus be reexpressed as

$x^{\prime}=x+M_{x}(s, t)$,

$y^{\prime}=y+M_{y}(s, t)$.

In the procedure used here a third-order polynomial is fitted to the mapping functions resulting ${ }^{8}$ in 20 coefficients in $s$ and $t$. This order polynomial is sufficient to remove even severe distortions. 


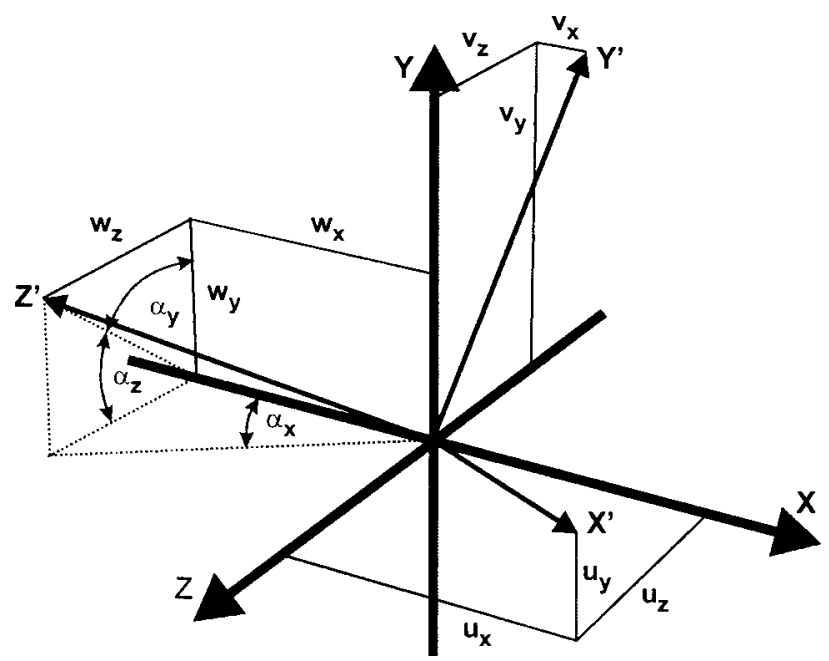

Fig. 2 Relationship of the experiment coordinate system $(X, Y, Z)$ and the view coordinate system $\left(X^{\prime}, Y^{\prime}, Z^{\prime}\right)$.

\section{View Angle Determination}

The true $(X, Y, Z)$ coordinate system aligned with the ROI and experiment can be represented by unit vectors $u$ $=(1,0,0), v=(0,1,0)$, and $w=(0,0,1)$. In the observed image these unit vectors are transformed to a camera coordinate system, as shown in Fig. 2, defined by

$u^{\prime}=\left(u_{x}, u_{y}, u_{z}\right)$,

$v^{\prime}=\left(v_{x}, v_{y}, v_{z}\right)$,

$w^{\prime}=\left(w_{x}, w_{y}, w_{z}\right)$

In the ideal case of looking perpendicular to the $X-Y$ plane, $u^{\prime}=(1,0,0), v^{\prime}=(0,1,0)$, and $w^{\prime}=(0,0,1)$. The observed components $u_{x}, u_{y}, v_{x}$, and $v_{y}$ are directly related to the local spatial derivatives of the mapping function via

$u_{x}=E_{x x} F$,

$u_{y}=E_{y x} F$,

$v_{x}=E_{x y} F$,

$v_{y}=E_{y y} F$,

where $F$ is a magnification factor, and

$$
\begin{aligned}
& E_{x x}=\delta M_{x}(x, y) / \delta x, \\
& E_{y x}=\delta M_{y}(x, y) / \delta x, \\
& E_{x y}=\delta M_{x}(x, y) / \delta y, \\
& E_{y y}=\delta M_{y}(x, y) / \delta y .
\end{aligned}
$$

The following definitions of a unit vector and the mapping of one orthogonal vector onto another are also valid:

$u_{x}^{2}+u_{y}^{2}+u_{z}^{2}=1$

$v_{x}^{2}+v_{y}^{2}+v_{z}^{2}=1$

$u_{x} v_{x}+u_{y} v_{y}+u_{z} v_{z}=0$.

The optical axis of the camera is aligned with the $Z^{\prime}$ axis and passes through the central pixel of the camera array and is described by the unit vector $\hat{o}$. The vector to be determined for each pixel $(i, j)$ in the array is then the vector $\hat{o}_{(i, j)}$, where the coordinate system has been translated so that $Z^{\prime}$ is originated at the pixel $(i, j)$. This new view vec-

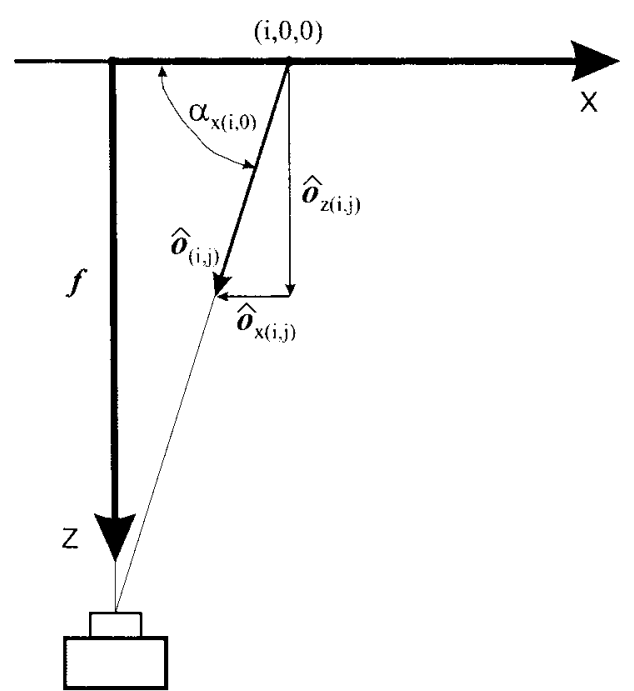

(a)

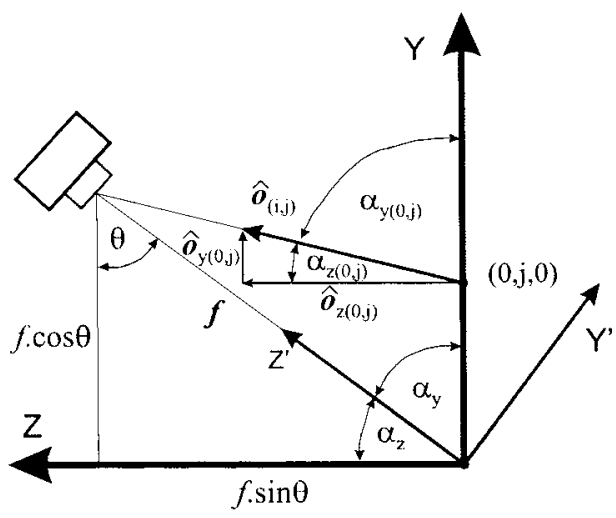

(b)

Fig. 3 Derivation of the components of $\hat{o}_{(i, j)}$ to the camera frame of reference $\left(X^{\prime}, Y^{\prime}, Z^{\prime}\right)$ and the experiment frame of reference $(X, Y, Z)$ : (a) the $z$ and $x$ components and (b) the $y$ and $z$ components. 
tor $\hat{o}_{(i, j)}$ is described in Fig. 3 and can be determined from the cross-product of the unit vectors describing the $X^{\prime}$ and $Y^{\prime}$ axes at point $(i, j)$;

$$
\begin{aligned}
\hat{o}_{(i, j)} & =\left[w_{(i, j)_{x}}, w_{(i, j)_{y}}, w_{(i, j)_{z}}\right] \\
& =\left[u_{(i, j)_{x}}, u_{(i, j)_{y}}, u_{(i, j)_{z}}\right] \times\left[v_{(i, j)_{x}}, v_{(i, j)_{y}}, v_{(i, j)_{z}}\right] .
\end{aligned}
$$

The direction cosines of $\hat{o}_{(i, j)}$ are $\left[w_{(i, j)_{x}}, w_{(i, j)_{y}}, w_{(i, j)_{z}}\right]$. Three angles shown in Figs. 2 and 3 can now be defined to describe the view direction of an individual pixel located at $(i, j)$ in the CCD array by

$\alpha_{x}=\arccos \left(w_{x}\right)$,

$\alpha_{y}=\arccos \left(w_{y}\right)$,

$\alpha_{z}=\arccos \left(w_{z}\right)$,

\subsection{Solving the Equations}

Equations (6) to (9) and (14) to (16) are a set of seven equations for the seven unknowns $u_{x}, u_{y}, u_{z}, v_{x}, v_{y}, v_{z}$, and $F$. Eliminating $F$ via

$F=v_{y} / E_{y y}$,

gives

$u_{x}=a v_{y}$,

$u_{y}=b v_{y}$,

$v_{x}=c v_{y}$,

with

$a=E_{x x} / E_{y y}$,

$b=E_{y x} / E_{y y}$

$c=E_{x y} / E_{y y}$.

For only slight distortions $E_{x x}, E_{y y}$, and $a$ are around 1, while the shear terms $b$ and $c$ are very small. For the sake of simplicity we can also assume that $E_{x x}$ and $E_{y y}$ are positive and nonzero ( $x$ and $y$ axis are not inverted and not rotated through $90 \mathrm{deg}$ ).

Solving the equations leads to

$u_{z}^{2}=0.5 d \pm\left(e+0.25 d^{2}\right)^{1 / 2}$,

$v_{y}^{2}=\left(1-u_{z}^{2}\right) /\left(a^{2}+b^{2}\right)$,

$v_{z}^{2}=1-\left(c^{2}+1\right) v_{y}^{2}$,

$w_{z}=u_{x} v_{y}-u_{y} v_{x}$

with

$$
\begin{aligned}
d & =\left[(a-b c)^{2}-(a c+b)^{2}-\left(a^{2}+b^{2}\right)^{2}\right] /(a-b c)^{2} \\
& =1-e-\left(a^{2}+b^{2}\right)^{2} /(a-b c)^{2},
\end{aligned}
$$

and

$e=(a c+b)^{2} /(a-b c)^{2}$.

\subsection{Restrictions and Simplifications}

A unique choice needs to be made for the model to decide the direction sign of $u_{z}$ derived from Eq. (28) to be either positive or negative. The same is true for $v_{y}$ and $v_{z}$ from Eqs. (29) and (30). This choice relates to the two possibilities whether the top surface of the image is tilted into the negative or positive $Z$ space. There is no way to decide based on the local derivatives of the mapping function what sign the angle $\alpha$ should take.

For example, consider the camera viewing the image plane perpendicular, which is only true for some point in the middle of the image. Dividing the image into top and bottom halves, in the upper half of the image, the camera is viewing the plane with some-let us say, by definitionpositive angle $\left(+\alpha_{y}\right)$ toward the $Y$ axis. From the perspective distortion, the image appears to be tilted away from the CCD. Similarly, the bottom part of the image appears also to tilt away from the CCD with however a negative angle $\alpha_{y}$. The top half of the image has positive $\alpha_{y}$ and the bottom half a negative $\alpha_{y}$. Theoretically one could think of a saddle-like distortion, for which $\alpha_{y}$ is zero in the middle of the image and being positive (or negative) on one side with the surface being tilted in the same direction. In this case, the magnification factor would increase monotonically from the top to the middle of the image, and then decrease toward the bottom of the image.

To derive a solution, different potential outcomes need to be considered in solving for the unknowns. This particularly relates to Eq. (28), where there are several solutions that have different interpretations. Here, imaginary solutions are not considered.

\subsubsection{Case 1: $e=0$}

In Eq. (28), if $e=0$, both the positive and the negative square-root solution can be considered. If $e>0$ only the positive sign is allowed. Consider first the case $e=0$. This means that

$a c+b=0$,

which corresponds to

$E_{x x} E_{x y}+E_{y y} E_{y x}=0$.

This corresponds to a simple $Z$ axis rotation of the recorded image plane together with either a rotation around the $X$ axis or around the $Y$ axis. For the simplest case,

$b=E_{y x} / E_{y y}=0 \quad$ and $c=E_{x y} / E_{y y}=0 \rightarrow e=0$.

Either the $X$ axis or the $Y$ axis is compressed corresponding to a rotation around the $Y$ axis or the $X$ axis, respectively. 


\subsubsection{Simplified case 1-A: $b=c=e=0$, positive square-root solution to Eq. (28)}

The positive square-root solution in Eq. (28) is

$u_{z}^{2}=d=1-a^{2}$,

which requires

$a=E_{x x} / E_{y y}<1$,

and the unity vectors are given by

$u^{\prime}=\left[a, 0,\left(1-a^{2}\right)^{1 / 2}\right]$,

$v^{\prime}=(0,1,0)$

This corresponds to a simple rotation around the $Y$ axis, compressing the $X$ axis $(a<1)$.

\subsubsection{Simplified case 1-B: $b=c=e=0$, negative square-root solution to Eq. (28)}

Similarly, this case leads to

$u^{\prime}=(1,0,0)$

$v^{\prime}=\left[0,1 / a,\left(1-1 / a^{2}\right)^{1 / 2}\right]$,

and

$a=E_{x x} / E_{y y}>1$.

This corresponds to a simple rotation around the $X$ axis, compressing the $Y$ axis $(a>1)$.

\subsubsection{General case 1}

The general case $e=0$ with $E_{y x} \neq 0$ and $E_{x y} \neq 0$ means that the image plane is rotated in-plane around the $Z$ axis and the positive and negative square-root solution corresponds to an additional rotation around the $X$ or $Y$ axis, correspondingly.

\subsubsection{Case 2: $e \neq 0$}

As derived, only the positive square-root solution is valid:

$u_{z}^{2}=0.5 d+\left(e+0.25 d^{2}\right)^{1 / 2}$.

All other quantities are derived as given by Eqs. (29) to (33). This is the most general case with a rotation in-plane and a rotation around the $X$ axis as well as the $Y$ axis.

\subsection{Determination of the Sign of the View Angle}

Using the definitions given in Eqs. (18) to (20) the view vector and hence the view angle calculation procedure will not determine the sign of the view angle. If, however, only the derived direction cosines are considered and some simple rules are applied, then direction definitions can be generated. The rules in this case are derived from the view vector that is of interest. The vector direction is from a point on the target towards the camera in a coordinate system that is based on the target at the point of interest. At the origin of the target the view vector $\hat{o}_{(0,0)}$ $=\left[\hat{o}_{x(0,0)}, \hat{o}_{y(0,0)}, \hat{o}_{z(0,0)}\right]$ is aligned with the $Z^{\prime}$ axis and is described by $\left(w_{x}, w_{y}, w_{z}\right)$. At a point of interest in the image the vector pointing to the camera can be defined as, $\hat{o}_{(i, j)}$, where the direction cosines are $\left[\hat{o}_{x(i, j)}, \hat{o}_{y(i, j)}, \hat{o}_{z(i, j)}\right]$.

A conceptual picture of how the direction cosines would vary across an image can be developed. Consider a pure positive rotation about the $X$ axis. An image of the values of component $\hat{o}_{x}$ covering the ROI would have zero magnitude along the $Y$ axis, i.e., $\hat{o}_{x(0, j)}=0$. For the positive region of the $X$ axis, $\hat{o}_{x(+i, j)}$ would increase in magnitude and have a negative direction, as depicted in Fig. 3(a), where the $Y$ axis is out of plane. For the negative region of the $X$ axis, $\hat{o}_{x(-i, j)}$ would also increase in magnitude and have a positive direction. For a large angle of rotation about the $X$ axis, the magnitude of the vertical component $\hat{o}_{y}$ would increase from the top to the bottom of the image and its sign would be constantly positive over the whole image, as shown in Fig. 3(b).

A method to determine the sign has been developed based on determining the local partial derivative of the

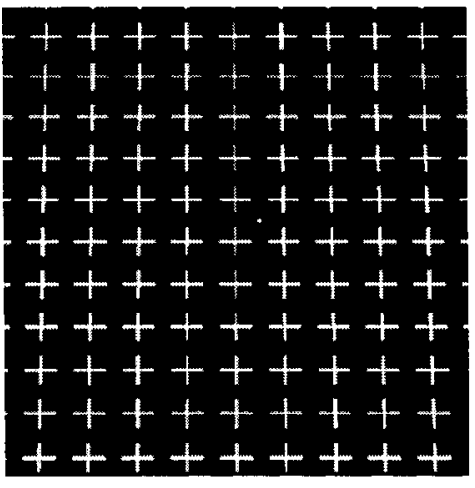

(a)

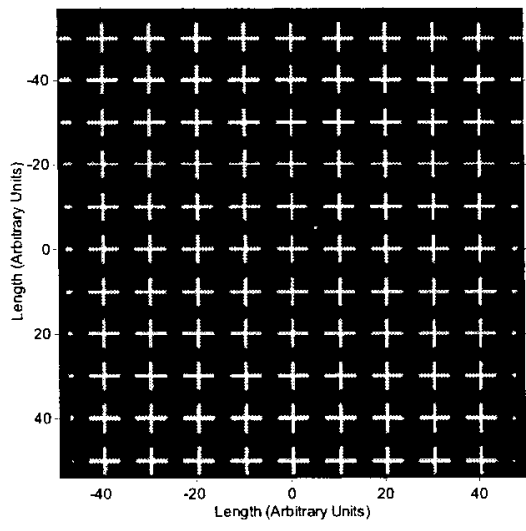

(b)

Fig. 4 Computer-generated image of a target of coplanar location markers viewed with a 30-deg rotation about the $X$ axis: (a) the raw image and (b) the dewarped and scaled image. 


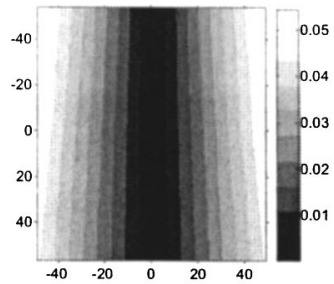

(a)

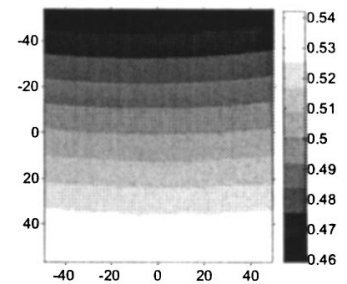

(b)

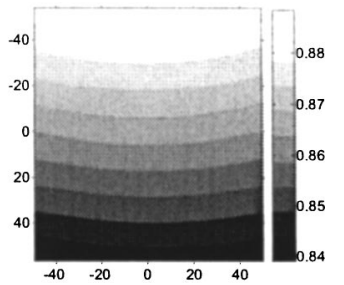

(c)

Fig. 5 Distribution of the magnitude (gray scale) of the direction cosines: (a) $\hat{o}_{(i, j) x}$, (b) $\hat{o}_{(i, j) y}$, and (c) $\hat{o}_{(i, j) z} \cdot$ (Axes in arbitrary length units.)

magnitude of the local direction vector component. Using the conceptual picture of the direction vectors for a pure rotation about the $X$ axis, the $X$ direction vector would go from positive to negative for the left-to-right regions of the image. This would match the sign of the local partial directive in the horizontal direction. The local partial derivative can be determined using a horizontal Sobel filter on the image. The sign of this derivative can then be used to define the sign of the direction vector. A similar procedure can be developed for the vertical component. Since the $Z$ axis has been defined earlier as pointing toward the camera, the $w_{z}$ component of the local view vector will always have a positive direction.

\section{Results and Discussion}

To test the procedure an image of a test target consisting of a regular array of coplanar crosses was generated using a 3-D drawing package. ${ }^{9}$ This allowed manipulation of the view of the target to a know angle. Camera parameters, including the position of the camera and camera view orientation can be explicitly included into the target model. The package also enabled lens modeling of the view to generate perspective distortion using a pin-hole camera model. An image of the target with crosses spaced at a known regular interval is shown in Fig. 4(a). For this image, the target has been rotated about the $X$ axis by $30 \mathrm{deg}$ with the top of the target tilted toward the camera. To generate the target, dimensionless length units in a ratio that might be typical of an experiment where used. Arbitrary units are used as length scales. The camera is located at $P_{\text {cam }}=(0,0,1000)$ in the reference frame of the experiment and views a region $100 \times 100$ units. The view is generated by modeling a lens with a focal length of 250 units. The crosses are at a regular spacing in the $X$ and $Y$ directions of 10 units and have a line aspect ratio of $6: 1$. A significant advantage of computer generating the image is that there is no noise in the image and the image is in perfect focus.

Figure 4(a) shows an image of the perspective view of the target. In Fig. 4(b) the image has been dewarped and spatially scaled using the information derived from the dewarping mapping functions. The image has increased in size (total number of pixels) to accommodate all of the original data that was captured and shown in Fig. 4(a). At locations outside the original data the image has been padded with intensities of zero value. The image in Fig. 4(b) shows that the dewarping algorithm has effectively stretched the image in the $Y$ direction.

Using the technique outlined earlier the spatial variation in the mapping function has been used to determine the direction cosines of the vector $\hat{o}_{(i, j)}$ at each pixel location $(i, j)$ in the image. Maps, the same size (number of pixels) as the dewarped image, of the magnitude of the components of the direction cosines are shown in Fig. 5. The derivation of the direction cosines based on the spatial gradient of the mapping function does not determine the sign of the individual components, only the magnitude, so only the absolute values are shown in Fig. 5. Using the rules discussed earlier, the sign of the components can be determined and are shown in Fig. 6. We can see in Fig. 6 that for the case of a pure rotation about the $X$ axis only, the sign direction of the $\hat{o}_{x(i, j)}$ component is altered.

With the sign and magnitude of the direction cosines determined it is a simple process to calculate the related angles. Maps of these angles, as defined in Figs. 2 and 3, are shown in Fig. 7. The conceptual picture of the variation in view direction angle discussed earlier can be seen in Fig. 7. The component $\hat{o}_{x(i, j)}$, expressed as the angle $\alpha_{x}$ in Fig. 7 (a), shows that at $i=0$ the view angle, in the $X$ direction, is perpendicular to the plane of the target. Away from this point $\hat{o}_{x(i, j)}$ increases in magnitude and at the extent of the

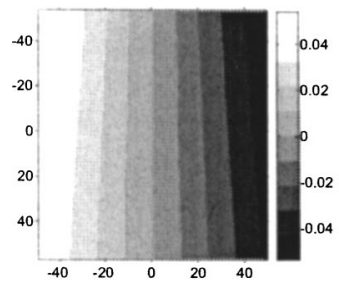

(a)

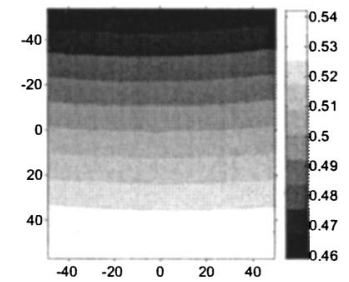

(b)

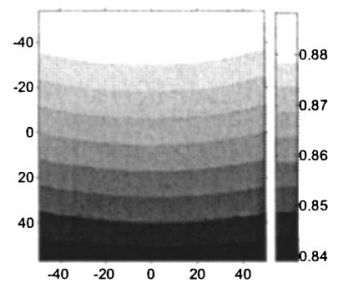

(c)

Fig. 6 Distribution of the magnitude (gray scale) of the signed direction cosines: (a) $\hat{o}_{(i, j) x}$, (b) $\hat{o}_{(i, j) y}$, and $(\mathrm{c}) \hat{o}_{(i, j) z} \cdot$ (Axes in arbitrary length units.) 


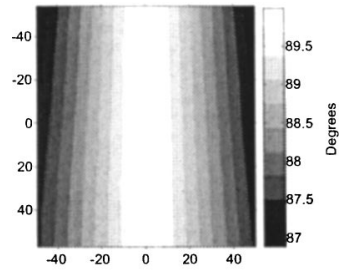

(a)

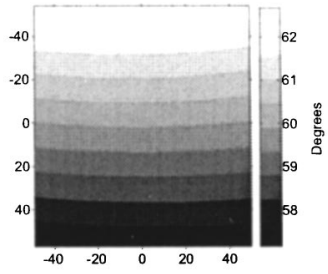

(b)

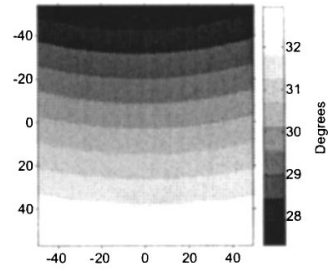

(c)

Fig. 7 Distribution of the related angles of the direction cosines: (a) $\alpha_{x}$, (b) $\alpha_{y}$, and (c) $\alpha_{z}$. (Axes in arbitrary length units.)

view a variation in the view angle of $\sim 6 \mathrm{deg}$. The angles $\alpha_{y}$ and $\alpha_{z}$, as shown in the Figs. 7(b) and 7(c), are uniquely related on the $Y$ axis by $\alpha_{x}=90 \mathrm{deg}-\alpha_{z}$. Angle $\alpha_{z}$ shows directly the magnitude the rotation applied to the test target. At the center of the image the rotation is measured as $30 \mathrm{deg}$, equal to the bulk view angle used to generate the target model. Away from the center of the image in the $X$ direction there is variation of the angle due to the bulk rotation applied and to perspective distortion. At the limits of the image the variation in the measured rotation of the test target is $\sim 6 \mathrm{deg}$.

A simple pin-hole camera model ${ }^{7}$ of the view of the target can be used to investigate the accuracy of the determined view angle along the major axes of the image. Using the definitions shown in Fig. 3, the depicted angles can be calculated along the major axis using the following:

$\alpha_{x(i, 0)}=\tan ^{-1}\left(\frac{f}{i}\right)$,

$\alpha_{y(0, j)}=\tan ^{-1}\left(\frac{f \cos (\theta)}{f \sin (\theta)-j}\right)$

$\alpha_{z(0, j)}=\tan ^{-1}\left(\frac{f \sin (\theta)-j}{f \cos (\theta)}\right)$

Here, the bulk view angle $\theta$ is the angle of rotation about the $X$ axis and is equivelent to the angle that is made with the $Z$ axis $\alpha_{z}$. Several values of $\theta$ in steps of $10 \mathrm{deg}$ were used in this test from $\theta=0 \mathrm{deg}$, which is a perpendicular view of the target, to $\theta=60 \mathrm{deg}$, which is near the practical extent of what would be used in an experiment. The variation of $\alpha_{x}, \alpha_{y}$, and $\alpha_{z}$ in degrees along the major axes is shown in Fig. 8 for these test cases. The procedure to determine the view shows excellent agreement with the simple pin-hole camera model of view angle components for a few bulk angles in the range 10 to $60 \mathrm{deg}$. There is some variation in the gradient seen in the plot, however, the error in the difference between the two results is less than $0.75 \%$. The technique breaks down for bulk view angles that are below $10 \mathrm{deg}$, which are views that are close to a perpendicular view. This is particularly highlighted in Fig. 8(a) where the measured angle for all cases except for $\theta=0$ deg is similar. In the experiment, the views used in PDV are often away from the perpendicular in the range 20 to 50 deg. Hence in practical use, the technique will resolve the view angle for each individual pixel in the view.

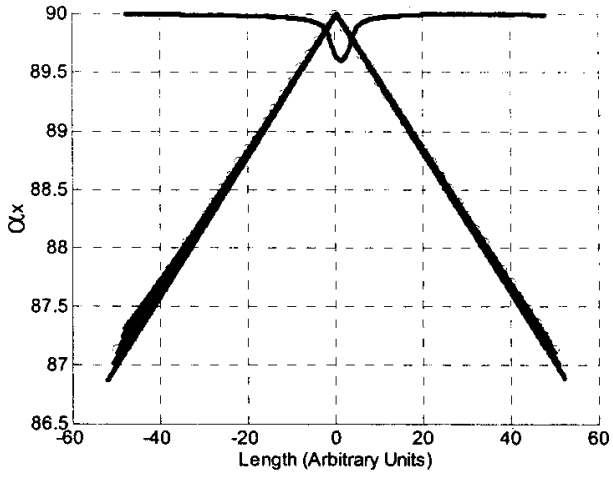

(a)

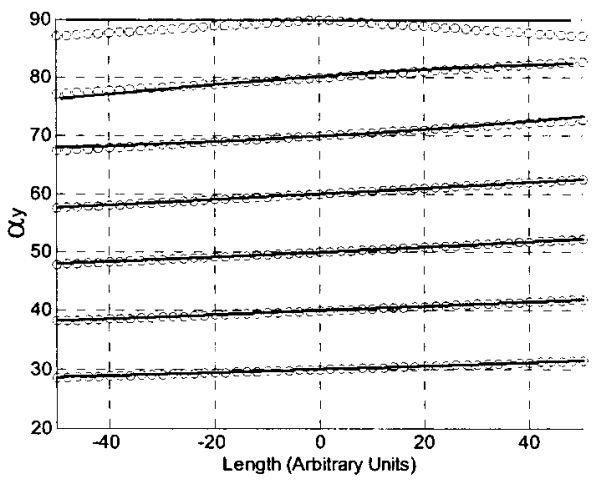

(b)

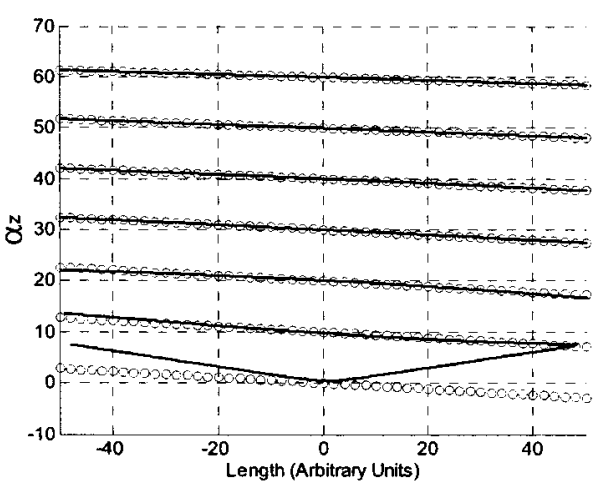

(c)

Fig. 8 Distribution of the calculated view angle $(\bigcirc)$ compared to the angle determined using a pin-hole camera model (-): (a) angle $\alpha_{X}$ along the $X$ axis, (b) angle $\alpha_{y}$ along the $Y$ axis, and (c) angle $\alpha_{z}$ along the $Z$ axis. (Abscissa in arbitrary length units.) 
The technique relies on the accurate determination of the mapping functions used to map the warped image onto a regular grid. Any degradation of the target image by noise, blurring of the image, or poor contrast will affect the performance of the dewarping technique in accurately determining the location of the markers. This will lead to an error in the mapping function and hence to an error in the determination of the view angle.

\section{Conclusions}

A procedure for determining the view vector of each individual pixel in a CCD array from a known planar target was developed. The procedure uses the spatial gradient in the mapping functions used to dewarp the image to determine the direction cosines from the viewed region to the individual capture pixel in the camera array. The procedure requires that a known target of coplanar location markers is used to enable determination of both the mapping functions as well as scaling parameters of the image. The known scaling of the location markers is all that the procedure requires to determine view direction cosines. No other physical measurement is necessary. This procedure is shown to give results that enable correction for individual pixel view vectors, while determining the bulk view angle to the test target. This procedure shows that accurate determination of view vector can be achieved without physical in situ measurement making the procedure useful for situations of confined access or where the range of the view is small.

\section{Acknowledgments}

The work was funded by the Engineering and Physical Sciences Research Council (EPSRC), United Kingdom.

\section{References}

1. J. F. Meyers and H. Komine, "Doppler global velocimetry: a new way to look at velocity," Laser Anemomet. 1, 289-296 (1991).

2. D. S. Nobes, H. D. Ford, and R. P. Tatam, "Three component planar Doppler velocimetry using imaging fibre bundles," in Proc. 11th Int Symp. on Applications of Lasers Techniques to Fluid Mechanics, Lisbon, Portugal paper 9-5 (2002).

3. R. Jones and C. Wykes, Holographic and Speckle Interferometry, (Cambridge University Press, Cambridge, 1989).

4. Y. Y. Hung, "Shearography: a new optical method for strain measurement and nondestructive testing," Opt. Eng. 21(3), 391-395 (1982).

5. A. Gruen and T. S. Huang, Eds., in Calibration and Orientation of Cameras in Computer Vision, Springer-Verlag (2001)

6. R. Y. Tsai, "A versatile camera calibration technique for highaccuracy 3D machine vision metrology using off-the-shelf TV cameras and lenses," IEEE J. Robot. Automat. RA-3(4), 323-344 (1987).
7. Z. Zhang, "A flexible new technique for camera calibration," IEEE Trans. Pattern Anal. Mach. Intell. 22(11), 1330-1334 (2000).

8. S. M. Soloff, R. J. Adrian, and Z. C. Liu, "Distortion compensation for generalized stereoscopic particle image velocimetry," Meas. Sci. Technol. 8, 1441-1454 (1997).

9. Rhinoceros "NURBS modelling for windows," http:// www.rhino3d.com/.

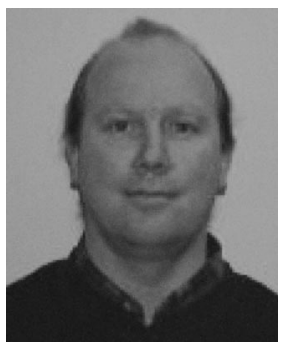

David S. Nobes joined the School of Engineering at Cranfield University, United Kingdom, in 1999. Previously, he was with the Department of Mechanical Engineering at the University of Adelaide, Australia, where he worked on the fluid dynamic and combustion problems of industrial scale gas burners. He was also involved in industrial scale burner trials at the International Flame Research Foundation (The Netherlands) and burner commissioning within cement kilns. Dr. Nobes was part of the research team that developed the Olympic relay torch for the Sydney 2000 Olympic, Games and conducted the initial development trials on the burner used for the stadium. At Cranfield University, he has been working on the development of planar Doppler velocimetry instrumentation for flow measurement applications within gas turbine compressors and wind tunnels.

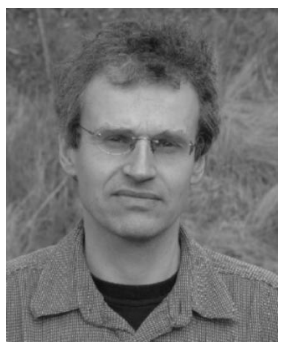

Bernhard Wieneke received his MS in astrophysics at Rice University, Houston, Texas. Since 1989, he has been working with LaVision $\mathrm{GmbH}$ developing hardware and software for laser and CCD-based optical measurement systems. His special interests are advanced PIV algorithms.

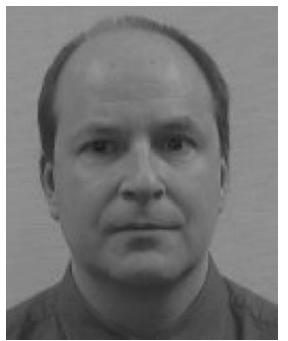

Ralph P. Tatam is a professor of engineering photonics in the School of Engineering at Cranfield University, United Kingdom, and heads the Optical Sensors Group. He has been active in optical and optical fiber based instrumentation and sensing for nearly 20 years. His current research interests include optical flow measurement, speckle interferometry, and optical fiber sensors. He graduated with a BSc in physics and chemistry from Exeter University, United Kingdom, in 1981, and received a PhD in physics Council for National Academic Awards in 1986. Following four years at the Physics Laboratory at the University of Kent he moved to Cranfield University. He has published more than 250 papers and has been involved with numerous national and international conferences. 